\title{
Erratum: Spatial applications of topological data analysis: Cities, snowflakes, random structures, and spiders spinning under the influence [Phys. Rev. Research 2, 033426 (2020)]
}

\author{
Michelle Feng (을 and Mason A. Porter (1) \\ (Received 23 April 2021; published 18 May 2021)
}

DOI: 10.1103/PhysRevResearch.3.029003

Synthetic networks. In Sec. III A of our paper, the image in Fig. 5 is not the one that we intended. We show the correct image in Fig. 1 of this erratum. The image in Fig. 5 in the paper is an example that uses the same dynamical process and family of networks, but it is for an instantiation of the process with $\rho=0.1$. The correct version of Fig. 5 is from a simulation with $\rho=0.05$ and corresponds to the example that we showed in Fig. 4(a).

Additionally, in Fig. 4, the specific numerical values that are next to the color bar apply only to panel (c). The qualitative meaning of the colors applies to all panels.

Finally, we clarify the following sentence about Watts-Strogatz (WS) networks: "In Fig. 7, we see that a WS small-world network also eventually has an infection network that consists of a single connected component." This sentence describes a true feature of our simulation, but the words "we see" imply that one can necessarily see this in the plot without ambiguity, and that is not the case.

Clarification of our procedure for clustering persistence diagrams (PDs). In our hierarchical clustering of PDs, we calculate pairwise distances between PDs of $H_{1}$ features only. This is the default choice in the software package GUDHI.

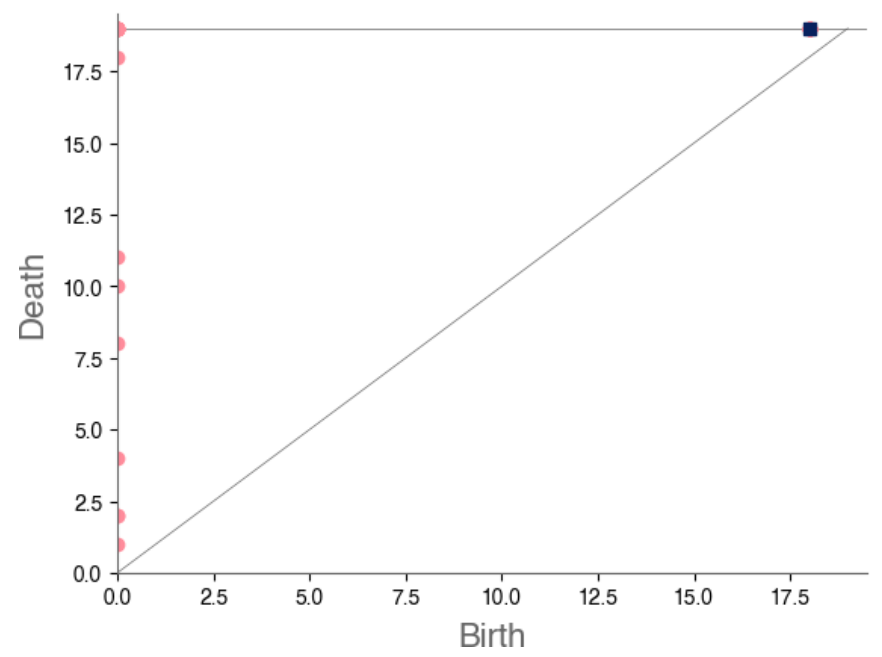

FIG. 1. The persistence diagram (PD) of an instance of the Watts threshold model on a random geometric graph. This figure corrects Fig. 5 of our paper.

Published by the American Physical Society under the terms of the Creative Commons Attribution 4.0 International license. Further distribution of this work must maintain attribution to the author(s) and the published article's title, journal citation, and DOI. 